\title{
Dynamics of a flexible tethered satellite system utilising various materials for coplanar and non-coplanar models
}

\begin{abstract}
This paper discusses the development of mathematical models for a flexible tethered satellite system (TSS) in both planar and co-planar states. The flexible tethered satellite system consists of three rigid bodies with two flexible tethers, each connecting two rigid bodies with one located in the centre and serving as the mothership. The TSS motion includes tether deformations, rotational dynamics, and orbital mechanics. The three materials that are possible to be used for a space tether are tungsten wire, Spectra-2000, and diamond; it should be noted that the diamond used here is in a form of a nanotube thread. The tether will undergo a spinning motion as well in the motorised option. In addition, the air drag perturbation is also considered since the entire TSS is flown around the Low Earth Orbit (LEO), where the air-drag perturbation is dominant. A survival analysis was then performed for planar and non-coplanar models in order to establish a dynamic performance envelope with respect to the tether's tension at different altitudes under the air-drag perturbation. The proposed models were treated numerically and analysed accordingly. Then a comparison study between the coplanar and noncoplanar models were conducted and the difference in their performances was observed and discussed. Although all materials have their own safe operation boundaries, the flexible TSS using tungsten shows a better dynamic performance than the other TSS options in a noncoplanar model.
\end{abstract}

Keyword: Tether material; Coplanar model; Non-coplanar model; Air-drag perturbation 